FORMATION Formation emploi

Revue française de sciences sociales

115 | Juillet-Septembre 2011

Le développement de l'aide à la personne : quelle professionnalisation?

\title{
Les recompositions du travail d'aide à domicile en France
}

Restructuring the home care profession in France

Die Umstrukturierung der Arbeit in der Haushaltshilfe in Frankreich

Las recomposiciones del trabajo de ayuda a domicilio en Francia

\section{Emmanuelle Lada}

\section{(2) OpenEdition}

Journals

Édition électronique

URL : http://journals.openedition.org/formationemploi/3410

DOI : 10.4000/formationemploi.3410

ISSN : 2107-0946

Éditeur

La Documentation française

Édition imprimée

Date de publication : 23 septembre 2011

Pagination : 9-23

ISSN : 0759-6340

Référence électronique

Emmanuelle Lada, « Les recompositions du travail d'aide à domicile en France », Formation emploi [En ligne], 115 | Juillet-Septembre 2011, mis en ligne le 03 octobre 2013, consulté le 30 octobre 2020. URL : http://journals.openedition.org/formationemploi/3410 ; DOI : https://doi.org/10.4000/ formationemploi.3410

(c) Tous droits réservés 


\title{
DOSSIER
}

\section{Les recompositions du travail d'aide à domicile en France}

Emmanuelle Lada*

\begin{abstract}
Suite aux récentes orientations de l'intervention publique, on observe, au sein des associations d'aide à domicile, de nouvelles prescriptions du travail et des changements d'organisation qui sont en contradiction avec le travail effectif réalisé au domicile.
\end{abstract}

On observe en France, à l'instar d'autres pays européens (Bettio, Plantenga, 2004 ; Simonazzi, 2008 ; Degavres, Nyssens, 2008) et plus largement du Nord (Cloutier et alii, 2008 ; Ito, 2010), une transformation des régimes de care $^{l}$ et de l'action publique qui participe à les configurer. Les modalités et le calendrier de ce changement varient certes selon les contextes sociétaux ; cependant, ces pays sont tous confrontés à des mutations de l'organisation sociale, économique et politique de l'aide, du soin et de la dépendance, sous l'effet d'une part, d'une réduction des coûts des politiques sociales et, d'autre part, du vieillissement démographique.

L'aide à domicile rémunérée, destinée aux personnes âgées, est particulièrement concernée par cette dynamique. Ces dernières années, en France, comme dans d'autres pays, cela s'est traduit par une nette tendance

\footnotetext{
${ }^{1}$ Care regimes : that is, the way in which the financing and provision of care are organised in the various systems (...) (Simonazzi, 2008 , p. 22) (Traduction : « c'est-à-dire la façon dont le financement et la prestation (la délivrance) d'aide sont organisés au sein de différents systèmes $»)$.
}

à la marchandisation (Devetter et alii, 2008). Acteurs et modes de régulation, périmètre de l'aide à domicile ont ainsi connu de profonds changements depuis le début des années 2000, et plus encore à partir de 2005 . Ce processus, porté par les pouvoirs publics, a eu des conséquences sur les parcours professionnels des

* Emmanuelle Lada est sociologue, professeure-assistante à l'université de Lausanne (UNIL)/ Centre en Études Genre LIEGE de I'UNIL et Equipe Genre, Travail, Mobilités (GTM) du Centre de recherches sociologiques et politiques de Paris (CRESPPA)-CNRS. Ses travaux portent sur les transformations des normes d'emploi et du travail en bas de l'échelle des qualifications ainsi que sur les effets de ces transformations sur les parcours (professionnels, de formation, familiaux et de santél des salarié.e.s. Ces objets de recherche sont abordés dans une perspective de genre, articulée aux problématiques liées aux migrations (approches intersectionnelles). Dans ce cadre, I'auteur a mené des recherches sur l'aide à domicile. 
salariées de l'aide à domicile ainsi que sur leurs conditions de travail, comme le révèlent des recherches menées en France (Doniol-Shaw, Lada, Dussuet, 2007) ou dans d'autres pays (Aronson et alii, 1996 ; Cloutier et alii, 2008).

Dans ce contexte, et à la suite de ces derniers travaux, le présent article propose de s'intéresser à un objet qui constitue encore un angle mort de la recherche et du discours public en France : le travail d'aide à domicile et ses recompositions, sous l'effet du tournant pris, notamment à partir de 2005, par les politiques publiques de formalisation, de professionnalisation et de financement de l'aide à domicile rémunérée. L'analyse concerne plus particulièrement l'aide aux personnes âgées en milieu associatif qui constitue, en France, la forme majoritaire de l'aide à domicile : en effet, au-delà des discours mêlant aide aux enfants, aux personnes handicapées et âgées, l'aide aux personnes à domicile est dans les faits d'abord et surtout une aide aux personnes âgées, l'aide auprès des enfants étant extrêmement minoritaire. Le secteur associatif concentre toujours la très grande majorité des intervenantes travaillant sur le segment formel de l'aide à domicile auprès des personnes fragilisées (78 \%)
(Marquier, 2010). Notre choix a également été motivé par l'ampleur des réorientations que l'on y observe. À ce titre, dans le contexte étudié, où l'action publique et l'action associative sont historiquement imbriquées pour dessiner les contours de l'offre de soins et d'aide à domicile rémunérée (Dussuet, Loiseau, 2007), nous analyserons les recompositions en cours à la lumière de cette co-construction.

Nous présenterons d'abord les principales mesures qui ont organisé la mutation, au cours de la décennie, de l'aide à domicile, un domaine où les conditions de travail sont dessinées par les modes d'exercice de l'intervention à domicile et les acteurs qui les encadrent. Dans un second temps, nous soulignerons les traits saillants du travail réel des aides à domicile, à partir du cas de structures associatives relevant de l'une des principales fédérations françaises. Nous analyserons enfin les écueils des transformations en cours sur le travail et les pratiques des salariées. Nous nous appuyons ici sur les résultats d'une recherche qualitative, menée en 2007, en France, auprès de salariées expérimentées de l'aide à domicile, afin d'analyser leurs parcours (Doniol-Shaw, Lada, Dussuet, 2007).

\section{Encadré 1. \\ Terrain et méthode d'enquête}

Cette recherche, financée par le Service des droits des femmes, visait à comprendre comment, dans un univers structuré par un important turn-over et la précarité $\left({ }^{*}\right)$, des salariées prennent place dans les métiers de l'aide à domicile et quel était leurs parcours (familiaux, d'emploi, de (délqualification). Cinquante-cinq entretiens biographiques ont été réalisés auprès de femmes, d'origine différente, cumulant au moins cinq ans d'ancienneté dans l'aide à domicile et occupant diverses positions dans la hiérarchie des qualifications. Un temps des entretiens a été consacré à remplir un agenda hebdomadaire afin de reconstituer l'emploi du temps et le contenu du travail de la semaine précédent l'enquête de terrain, heure par heure. Ce dispositif d'enquête a été complété par des entretiens auprès d'encadrant.e.s, de responsables associatifs et d'acteurs publics ainsi que par une analyse documentaire. Ce volet de l'enquête a permis de relever des données sur l'histoire organisationnelle des associations enquêtées ainsi que sur les politiques et pratiques de gestion des personnels. Six associations ont été enquêtées, dans différentes régions françaises, tant rurales qu'urbaines. Elles salariaient de 50 à 400 personnes.

(*) : Cette précarité genrée prend des formes qui la rendent peu visible (Dussuet, 2005), comme l'informalité, les statuts d'emploi (l'emploi direct, par exemple). La précarité tient également à la faiblesse des durées de travail et à la faible qualification reconnue aux emplois ; ce qui, combiné avec les faibles durées travaillées, conduit à des rémunérations particulièrement basses, ne permettant guère l'accès à l'autonomie économique aux femmes concernées. Les associations enquêtées suivent toutes une politique d'augmentation des temps de travail et d'amélioration des conditions de travail. 


\section{LES ASSOCIATIONS D'AIDE À DOMICILE À L'ÉPREUVE DE L'INFLEXION DES POLITIQUES PUBLIQUES}

\section{Des recompositions continues}

En France, l'aide à domicile se caractérise par un mouvement de recompositions permanentes depuis les années 80 . De nombreux dispositifs publics se sont en effet juxtaposés ou succédés pour encadrer et formaliser ce segment du marché du travail, (Gaullier, 1986 ; Devetter et alii, 2008) précarisé et hyper féminisé dans les emplois d'exécution et d'encadrement intermédiaire. Rejoignant une dynamique transnationale de transformation des régimes de care qui se nourrit de la division sexuelle, sociale et ethnique du travail, ce mouvement s'est accéléré au début des années 2000, et à nouveau à partir de 2005 (Devetter et alii, 2008). Dans ce cadre, on a assisté à la mise en œuvre de nouvelles "politiques de prestations" (Jenson, 2001) de maintien à domicile, portées, d'un côté, par l'augmentation du poids sociodémographique des personnes de plus de soixante ans ; de l'autre, par la recomposition des politiques et de l'action publiques en faveur de la réduction des coûts de santé et de la dépendance ou en matière d'emploi, comme dans d'autres pays (Cloutier et alii, 2008).

\section{De la convention de professionnalité par la qualification...}

Dans les premiers temps, les mesures instaurées ont plus particulièrement actionné le levier de la formation et de la qualification des salariées et, en filigrane, celui des cadres du travail. Il s'agissait d'améliorer l'attractivité des métiers et, ce faisant, la qualité de l'offre d'aide à domicile. Ces mesures, que l'on doit également aux mobilisations syndicales et aux associations qui ont soutenu, voire impulsé, les orientations

\section{Encadré 2}

\section{L'aide à domicile rémunérée : une pluralité d'acteurs, des formes de mise au travail variables}

L'aide à domicile rémunérée n'est pas homogène. Ses segments formels comptent une pluralité d'employeurs, des particuliers aux établissements publics (via les CCAS - Centres communaux d'action sociale), en passant par les entreprises (Très Petites Entreprises, enseignes...) et les associations. Ces dernières forment elles-mêmes un paysage très diversifié, en termes d'organisation (recours ou non à des bénévoles, au seul mode mandataire, dans lequel l'employeur est la personne qui bénéficie de l'aide, ou combinaison du mandataire et prestataire), de statut (associations intermédiaires, fédérées ou non...), d'offre de services, etc.

Dans cet univers pluriel, les formes sociales de mise au travail, les conditions de travail, voire le contenu du travail, peuvent varier, y compris au sein d'une même structure et pour une même salariée. Cette variabilité tend à s'organiser autour d'un fil conducteur. En effet, à la différence d'autres univers professionnels précarisés, cette variabilité relève moins du statut de l'emploi occupé (Lada, 2009) que des modes d'exercice de l'aide à domicile et des acteurs qui les organisent. Par exemple, les salariées intervenant en mode mandataire ou prestataire relèvent de conventions collectives différentes, quand elles existent, puisqu'à ce jour, les conventions collectives encadrant l'aide à domicile ne s'appliquent pas aux entreprises. Ce point est important car le mode mandataire (dans ce cas, c'est la convention collective des employeurs particuliers qui s'applique) tend à favoriser l'individualisation des conditions de travail qui prévalent encore dans l'emploi direct (Le Feuvre et al., 2001). Par ailleurs, cette convention garantit des droits et des conditions de rémunération moindres que la convention de l'aide à domicile, appliquée par les associations pour leur activité prestataire. De plus, elle ne prévoit pas la prise en compte des temps de déplacement dans le temps de travail. 


\section{Encadré 3}

\section{Le recours massif aux migrations internationales féminines dans l'aide à domicile aujourd'hui en France : quelle réalité ?}

Une doxa s'est imposée, en France, dans le sillage des recherches anglo-saxonnes sur l'internationalisation et la marchandisation du care d'une part, les migrations féminines d'autre part et enfin, la division internationale du travail : le recours massif, si ce n'est exclusif, à des migrantes des pays " du Sud » ou " de l'Est», nouvellement arrivées dans ce pays, pour organiser le prendre soin des personnes dépendantes. Or, la mobilisation de ces recherches pour rendre compte des "régimes de care mixtes » (Simonazzi, 2008), comme celui de la France, nous semble problématique. Et ce, d'autant plus si on n'adjoint pas à cette grille d'analyse les notions de division sociale, ethnique et sexuelle du travail.

Ces recherches permettent, certes, d'éclairer l'organisation de certains segments, notamment informels, de l'aide à domicile rémunérée dans les territoires les plus urbains et de rendre compte de la réalité vécue par ces migrantes. Elle occulte cependant la position occupée par d'autres catégories sociales de femmes dans l'aide à domicile, en général, et l'aide auprès des personnes adultes dépendantes, en particulier : les femmes de milieux populaires sans histoire familiale marquée par des migrations internationales mais aussi les femmes venues des départements et territoires-d'Outre mer ou encore les descendantes de migrant.e.s.

Une enquête très récente (Marquier, 2010) invalide la vision d'une sur-représentation des femmes migrantes étrangères dans l'ensemble des univers composant l'aide à domicile rémunérée en France. Elle nourrit également notre hypothèse de la relégation des femmes migrantes racisées (non blanches) aux marges et dans les positions subalternes de l'aide à domicile, ou encore de la variabilité des conditions de travail, selon la position occupée par les femmes dans la division sociale et ethnique. Si le critère de nationalité ne permet pas de saisir les dynamiques discriminatoires, il constitue néanmoins un premier outil fort utile. Il apparaît ainsi que seules $3 \%$ des intervenantes à domicile auprès d'adultes fragilisés sont étrangères.

publiques, sont importantes. Elles ont en effet porté la formation de groupes professionnels, tout en offrant de nouvelles perspectives en termes de droit du travail, de conditions de travail, de reconnaissance professionnelle ou de parcours professionnels (Doniol-Shaw, Lada, Dussuet, 2007). Ainsi, la reconnaissance des acquis de l'expérience, la création du diplôme d'État d'auxiliaire de vie sociale (DEAVS), accessible par le biais de la formation initiale et non plus ouvert qu'à des personnes en emploi, (comme c'était le cas du CAFAD - Certificat d'aptitude aux fonctions d'aide à domicile - qu'il remplace), l'accord de branche en 2002 sur les emplois et les rémunérations revalorisant en particulier les métiers de l'intervention à domicile, ont ainsi ouvert de nouveaux possibles en termes de carrière et de reconnaissance des savoirs et savoirfaire. L'accord de branche, propre aux seules associations, a quant à lui permis de définir les profils de postes en rapport avec les qualifications, en les accom- pagnant d'une revalorisation sensible des rémunérations, notamment pour les titulaires du DEAVS.

La réforme plus globale des institutions sociales et médico-sociales dans laquelle s'inscrivaient ces mesures a alimenté ce mouvement. Elle a en effet soutenu la reconnaissance de la fonction sociale de l'aide à domicile, en élevant les exigences attendues des services et en se doublant de l'application d'une tarification individualisée par service (décret du 22/10/2003) prenant en compte les coûts réels et les niveaux de qualification des personnels. Enfin, la création d'un nouveau mode de financement de l'aide à domicile, l'APA (Allocation personnalisée d'autonomie), a participé de cette volonté politique de soutenir la reconnaissance des qualifications ainsi que l'amélioration des conditions de travail et de rémunération. La création de l'APA s'est accompagnée de règles de valorisation tenant compte des conventions collectives, tout en pénalisant le recours à une salariée 
en emploi direct sans expérience ou qualification et en orientant les personnes classées en GIR 1 et $2^{2}$, isolées ou nécessitant une surveillance régulière, vers les services prestataires.

Ces outils, qui couvrent le périmètre associatif, ont donc plus particulièrement été favorables aux salariées des associations (Marquier, 2010).

La professionnalisation des structures employeurs et de l'offre d'aide a également été visée (Ribault, 2008). La production de la norme des services à domicile NF X 50 O56 en $2000^{3}$ puis en 2004, la publication des règles de certification, sont significatives de ce mouvement. Ces dispositifs ont accéléré ou engagé des transformations dans des associations sous tensions (Prouteau, 2003). D'ailleurs, un certain nombre d'entre elles avait déjà amplifié et diversifié leur offre de service, tout en recourant à des outils importés de l'univers productif, l'univers associatif connaissant en effet d'importantes mutations depuis plusieurs décennies :

« En 93-94, il y a eu la création d'un service d'aide à domicile en direction de personnes âgées. (...) Le conseil d'administration s'est dit : "Ce n'est pas logique qu'on continue de ne s'intéresser qu'aux familles. On a des compétences, un savoir-faire et puis, peut-être qu'il y a un marché". Déjà, à l'époque, cette notion existait au niveau du conseil d'administration. Alors, peut-être serait-il bon qu'on s'intéresse aux personnes âgées? (...) "On abandonne le secteur Familles au profit des personnes âgées!" ” (Une directrice d'association)

\section{... à la convention} de professionnalité marchande

La mise en œuvre du plan « Borloo », également appelé «plan de cohésion sociale », au milieu des années 2000, est venue soutenir cette dynamique. Elle a cependant aussi indéniablement institué de nouvelles

\footnotetext{
${ }^{2}$ La grille AGGIR évalue la perte d'autonomie des personnes de plus de 60 ans. Elle comporte six niveaux. Le GIR 1 correspond à une grande dépendance physique et psychique, alors que le GIR 6 renvoie à des personnes qui n'ont pas perdu leur autonomie pour les actes de la vie courante.

${ }^{3}$ Cette norme établit un socle commun d'engagements de service sur les prestations afin de faciliter, de maintenir et d'améliorer la vie à domicile. Elle a connu différentes révisions, dont la dernière en 2008 .
}

réalités qui ont profondément fragilisé ces nouveaux acquis. Avec ce plan, les pouvoirs publics ont ainsi affirmé la volonté d'ancrer ce secteur sur le segment concurrentiel du marché du travail et ce, alors que la demande solvable, elle, n'a pas suivi. Cette politique de changement de régulation marque un tournant : la " convention de professionnalité marchande " (Ribault, 2008) ${ }^{4}$ s'impose. Elle a pris la forme de logiques de mises en concurrence entre associations mais aussi entre les associations et les entreprises (auxquelles une nouvelle place est accordée), voire avec les employeurs particuliers. Dans ce cadre, un mouvement de concentration de certaines associations s'est opéré, par le biais de reprises d'activité d'associations et/ou de CCAS (Doniol-Shaw, Lada, Dussuet, 2007), sur fond de multiplication et de diversification de l'ensemble des acteurs en présence (ouverture récente aux structures commerciales,...).

" Dans notre département, il y avait 230 associations qui étaient agréées avant les lois Borloo. Aujourd'hui, il y en a 350, donc, $50 \%$ d'augmentation en peu de temps. L'augmentation de l'activité globale, c'est $2 \%$. (...) Donc, ça veut dire qu'il y a eu forcément une redistribution des cartes entre un certain nombre d'acteurs. Ça n'a pas été notre cas. Nous, on a continué à augmenter, mais ça n'a pas été le cas d'autres collègues, qui ont vu leur activité diminuer de $7 \%$. » (Un directeur d'association)

Cette politique a aussi impacté les politiques et pratiques associatives, comme dans d'autres pays européens (Degavres, Nyssens, 2008). Conjuguée à l'augmentation importante des bénéficiaires de l'APA, elle a remis en question le processus de formation et de qualification des salariées, engagé par les acteurs associatifs et appuyé par les pouvoirs publics euxmêmes. Dans un contexte de territorialisation des politiques publiques, les conseils généraux ne sont plus en capacité de faire face aux demandes d'APA, l'État diminuant aussi sa participation à ce financement, via la CNSA (Caisse nationale de solidarité pour l'autonomie) : la part de financement de l'APA des conseils

\footnotetext{
${ }^{4}$ Nous suivons Ribault (2008) et Jany-Catrice et al. (2009) dans leur emprunt à Boltanski et Thevenot de la notion de convention pour rendre compte, ici, des signes et des effets de l'élaboration d'un nouveau compromis en matière de professionnalisation sur le travail des salariées de l'aide à domicile.
} 
généraux sur leur propre budget est en constante augmentation et pèse sur les finances locales.

Sur le terrain, le processus engagé de qualification des salariées a été atteint par ce mouvement, la qualification apparaissant comme une variable à mobiliser pour diminuer le coût de l'aide. On pense également aux injonctions publiques de professionnalisation et de rationalisation, et à leurs effets sur le terrain : introduction et/ou accélération de la diffusion du management, transformation ou introduction de pratiques d'évaluation des structures et de leurs salariées, production de normes de qualité : ces outils et modèles importés tels quels du secteur marchand et concurrentiel, apparaissant ici comme référent, sont en effet élaborés à distance, voire en contradiction avec le travail réel, tel qu'il se fait et s'organise au domicile des personnes âgées. Ces changements ont eu des conséquences sur l'organisation du travail et les contours du travail mais aussi les temps de travail, ceux-ci apparaissant comme co-construits par les politiques des employeurs et de l'action publique (DoniolShaw, Lada, Dussuet, 2007). Pour saisir les effets de ces transformations, il faut rappeler les traits saillants du travail d'aide à domicile. Nous ne traiterons pas ici des contraintes et des pénibilités qui ont fait l'objet d'un grand nombre de travaux (Aronson, 1996 ; Le Feuvre et Martin, 2001 ; Rocher et Langevin, 2005 ; Avril, 2006 ; Cloutier et alii, 2008 ; Guichet et al., 2009) : pénibilité physique et psychique, confrontation avec la mort, pressions temporelles, fréquence des accidents du travail...

\section{LE TRAVAIL D'AIDE À DOMICILE : L'ICI ET LE MAINTENANT}

\section{Des contours instables?}

Les contours formels du travail des aides à domicile sont variables. Dans le contexte français, l'aide à domicile auprès des personnes âgées (60 ans et plus) est en partie ou totalement financée par l'APA (Allocation personnalisée d'autonomie) ; dès lors, l'élaboration, voire la révision du plan d'aide, dans le cadre de ce dispositif, contribue à construire le contenu formel du travail des aides à domicile. C'est à ce moment que sont définies les tâches que les aides à domicile auront à accomplir, en fonction notamment du degré de dépendance évalué par une grille standardisée (grille AGGIR) ainsi que, pour les associations, $\mathrm{du}$ « travail proscrit » (Jeantet, 2003). C'est en effet lors de la mise en place du plan d'aide que les attentes des particuliers sont formulées auprès des acteurs associatifs, mais aussi que ces derniers expliquent les limites qui ne peuvent être franchies, en termes de contenu et charge de travail.

Outre ces dimensions, qui participent à l'élaboration de la fiche de poste pour des interventions auprès d'une personne fragilisée, la qualification de l'emploi occupé (A, B ou C, du moins qualifié au plus qualifié) permet aussi de définir le contenu formel du travail, en raison de la division du travail qu'elle instaure entre salariées de l'aide à domicile. Ainsi, les AVS (auxiliaires de vie sociale), qui possèdent la qualification la plus élevée (soit en C), sont théoriquement les seules à pouvoir procéder à une aide à la toilette.

Dans les faits, ces limites volent en éclats. Le périmètre de l'activité des aides à domicile est particulièrement instable. L'organisation du travail et les contours du travail d'aide, tel qu'il se fait au domicile des autres ${ }^{5}$, sont en effet co-construits par une pluralité d'acteurs, depuis l'association, la/les salarié.e.s et la personne aidée, en passant par son entourage ou le voisinage. "La dernière fois, elle a voulu me faire nettoyer la cave. Mais j'ai pas voulu. Elle a dit qu'elle allait le dire à l'association. Moi, j'ai prévenu l'association. On m'a dit qu'il ne fallait pas laisser faire. Mais c'est vrai que je suis coincée. J'ai déjà une histoire avec une dame. (...) Tous les lundis, j'arrivais et il y avait plein d'assiettes. Elle m'a dit qu'elle avait reçu ses enfants. J'ai dit à la dame: "vous savez, il y a une limite de travail. Je suis là pour vous, pas pour les autres." Qu'est-ce que j'ai pas dit là... Elle m'a foutue à la porte! Il y a sa petite fille qui travaille ici. Elle est venue me voir: "Comment ça se fait que tu n'as pas voulu lui faire la vaisselle?" " (Mme U., 30 ans, en C, 10 ans d'ancienneté).

\footnotetext{
${ }^{5}$ Les enjeux diffèrent considérablement entre travailler à domicile chez soi (c'est le cas, par exemple, des assistantes maternelles) et travailler au domicile des autres. Or, dans les dispositifs et classifications des métiers des services à la personne, l'unité de lieu, le domicile, prévaut sans que ces différents sens et enjeux soient intégrés.
} 
Les analyses sur les emplois de services ont montré la place du client dans l'organisation formelle du travail et le travail ; ici, la particularité nous semble résider dans le périmètre potentiellement « infini » de personnes pouvant intervenir, dans les faits, dans la re-définition du contenu du travail et son organisation.

De telles réalités imposent aux salariées des re-négociations constantes pour contrer de telles variabilités. Elles conduisent également les salariées à réaliser, seules, un travail d'intermédiation, au sein d'une configuration plurielle d'acteurs. Cette situation se répète de foyer en foyer, puisque les salariées interviennent chez plusieurs particuliers. Dans notre enquête, les salariées rencontrées pouvaient cumuler jusqu'à dix domiciles par semaine.

Il faut en outre compter avec la frontière floue, dans la pratique, séparant les actes relevant des métiers de soins à domicile des métiers de l'aide à domicile. Selon les négociations établies avec les intervenant.e.s prodiguant des soins de santé à domicile, mais aussi selon l'état de santé des personnes aidées, les salariées auront par exemple à accomplir, ou non, certaines tâches (la toilette, donner des médicaments, poser des bas de contention...). L'évolution de la santé des personnes aidées interfère également sur le contenu du travail ; il en est de même, dans un tout autre registre, de la succession des dispositifs encadrant l'aide à domicile ou encore l'offre de soins et de personnel soignant sur un territoire donné.

\section{Des situations de travail} plus complexes auprès de personnes âgées fragilisées

Si les contours du travail sont variables et instables, le travail des intervenantes de l'aide à domicile n'est pas, pour autant, indéfinissable. En France, les pouvoirs publics ont choisi de renforcer une politique de soutien au maintien à domicile de personnes âgées ; cela a accru le nombre de personnes continuant à vivre chez elles, ainsi que la demande d'accompagnement et de soins (Espagnol et al., 2008). Ce mouvement, qui a lieu sur de fond de vieillissement démographique, s'est accompagné de la transformation des profils des publics bénéficiaires. La perte d'autonomie intervient aujourd'hui plus tardivement (Danet, Salines, 2008); dès lors, cette politique a permis le maintien à domicile de personnes plus isolées et surtout plus dépendantes, atteintes par des pathologies lourdes propres au grand âge, caractérisées par d'importantes déficiences cognitives ou physiques (maladies d'Alzheimer, Parkinson...).

Les situations de travail des aides à domicile auprès de ces populations deviennent ainsi de plus en plus complexes. Elles tendent à s'éloigner de celles rencontrées par la figure de l'aide ménagère (que certaines des femmes qui cumulent le plus d'ancienneté dans l'aide à domicile ont connues). En outre, les intervenantes à domicile travaillent seules (Avril, 2006) et surtout en situation de travail isolé (Doniol-Shaw, Lada, Dussuet, 2007).

\section{Des réajustements constants et une co-élaboration : «faire avec », «faire faire » et « anticiper pour autrui »}

L'objectif du travail d'aide aux personnes fragilisées par l'âge, la maladie ou le handicap est de contribuer à préserver, le plus longtemps et le mieux possible, une autonomie en déclin. Les salariées de l'aide à domicile interviewées prennent très au sérieux cet objectif. Il implique que leur activité de travail soit sans cesse interrogée par l'état de la personne et son devenir. Il s'agit d'évaluer les priorités, de définir ce qui peut, mais aussi ce qui doit être fait par la personne ; l'objectif étant de tenir le fil du travail d'aide et de ne pas verser du côté de la prestation de service standardisée, reproductible d'un foyer à un autre.

Pour ce faire, les connaissances que les salariées ont acquises (en formation, par auto-formation ou par expérience) en matière d'hygiène, d'alimentation, de soins quotidiens, de psychologie, de risques spécifiques à ces populations fragilisées, ou sur les pathologies rencontrées, sont ainsi constamment mises au service de cette évaluation centrée sur la personne : "L'après-midi, je vais chez Mme J qui est atteinte de la maladie d'Alzheimer. On fait de la stimulation, on marche. En ce moment, je ne fais pas beaucoup de ménage car je l'ai sentie descendre d'un pallier, j'ai pris l'initiative de prévenir les docteurs. Elle a besoin d'être bien stimulée car elle parle moins; alors je la stimule, je la fais parler. » (Mme S., 48 ans, en C, 5 ans d'ancienneté) 
C'est sur la base de cette évaluation que s'organise, sur le moment et au domicile, le travail. La question de l'adéquation du travail prescrit à l'objectif $\mathrm{du}$ travail centré sur la personne est toujours présente.

Réaliser cette adéquation suppose de l'attention et repose sur une élaboration ici et maintenant, constamment renouvelée car : "Chaque personne est différente et je suis différente chez chaque personne. C'est une responsabilité, il ne faut pas rater son coup. " (Mme T, 32 ans, en A, 11 ans d'ancienneté)

Mais cette attention exige du temps ; toutes le disent, les personnes âgées attendent beaucoup (Avril, 2006), comme le confie l'une des salariées interviewées : "Quand on intervient trois fois chez la même personne, on a un suivi, on peut s'organiser, faire les choses calmement. Quand on vient une fois par semaine, les gens attendent énormément. On est leur rayon de soleil, c'est vrai, mais ils attendent beaucoup. Une fois par semaine, ça passe vite, la semaine, entre-temps, elle est longue, et j'ai l'impression, c'est personnel, qu'il faut plus s'investir. » (Mme R., 27 ans, en B, 4 ans d'ancienneté)

Il faut également du temps pour qu'une personne qui, faute d'une aide, avait laissé son hygiène et sa santé se dégrader, accepte de se laver ou de se coiffer à nouveau.

L'extrait d'entretien suivant, représentatif des propos tenus par l'ensemble des salariées, montre que ces manières de faire s'inscrivent dans un apprentissage : « J'ai appris à faire les choses régulièrement et calmement, pas à $200 \%$. Les toilettes, ça ne sert à rien de foncer, après la personne glisse... Efficace mais dans le calme et si quelque chose n'est pas fait, et bien, ça reste. Il vaut mieux protéger la personne. C'est important aussi, il faut qu'ils gardent leur dignité. " (Mme E., 36 ans, en A, 5 ans d'ancienneté)

Soulignons enfin que répondre aux besoins singuliers d'une personne ou travailler à l'évaluation de ces besoins ne signifie pas se plier aux exigences ou attentes de la personne ou de son entourage. Il faut même parfois aller à l'encontre de celles-ci : «Il faut savoir s'imposer avec tact. Je n'ai pas voulu céder, je sentais bien que si je ne le faisais pas (convaincre une personne de se faire aider pour sa toilette), ce ne serait pas fait non plus. Il faut savoir prendre en charge ce que l'on a à prendre en charge. » (Mme E., 36 ans, en A, 5 ans d'ancienneté)

Le travail réalisé par les salariées n'est ainsi jamais construit d'emblée et de façon pérenne. Ceci d'autant plus que la santé est un processus et non un état; cela induit des réajustements constants des manières de faire des aides à domicile mais aussi du travail à faire auprès des personnes aidées. L'impression de routine qui peut transparaître à la lecture des « plannings » ou dans les premiers temps des récits de pratiques des salariées s'efface bien vite. Ainsi, à propos d'une personne atteinte de la maladie de Parkinson et qui avait des difficultés à se laver : «Et puis, quand vous rentrez dans une maison, vous vous rendez compte, il $y$ avait des odeurs. Après, ça s'est fait en discutant, ça ne s'est pas fait tout de suite. Quand j'ai compris que sa fille ne venait qu'une fois par semaine, je me suis dit que ce n'était pas possible. Il fallait le faire quoi (sa toilette). C'est vrai que, dans notre travail, il faut savoir gérer ce qui est prioritaire. La maison, le nettoyage, ça peut n'être fait qu'une fois par semaine, tandis que la toilette, ça doit être fait tous les jours. Ce qui est important, c'est que la personne soit bien ; la propreté corporelle et avoir à manger, c'est essentiel. » (Mme X, 42 ans, en A, 10 ans d'ancienneté)

Cette dimension de l' « ici et maintenant » et la nécessité de procéder à des ajustements dans le temps, foyer par foyer, relèvent également des situations de travail. Le travail d'aide à domicile place les salariées dans une position où elles partagent le plus intime de la vie des personnes, dans un contexte de fragilité et de déclin. Accompagner cette fragilité nécessite l'instauration d'une relation de confiance. Les salariés soulignent que cette relation de confiance ne peut obéir à une norme reproductible en l'état d'un domicile à un autre car il s'agit d'une relation, ce qui implique une co-élaboration. Dans cette relation d'aide, qui repose sur la notion du « faire avec » ou du « faire faire » et non du « faire à la place », le travail est en effet partagé et la coopération de la personne est absolument nécessaire : "Il faut entrer dans leur mentalité, il faut entrer dans leur jeu..., pour que, la personne, elle ait envie de travailler avec vous. (...) Les doigts d'une main, ils ne sont pas pareils. Et bien, chaque personne âgée, c'est pareil. Il faut changer à chaque fois. » (Mme Z, 54 ans, en A, 16 ans d'ancienneté) 
Cela peut aller jusqu'à agir selon une logique qu'un regard extérieur, hors du contexte, pourrait qualifier de « faute professionnelle » : « Je lui ai dit quej'allais chercher le vin si je faisais le ménage. Pas de ménage, pas de vin. »C'est pourtant par cette voie que cette salariée a réussi à sortir de l'extrême clochardisation cet homme chez qui plus personne ne voulait venir (Doniol-Shaw, 2009) : " Maintenant, il remarche! Je lui ai fait avoir un déambulateur. Maintenant, je lui dis que, s'il marche sans son déambulateur, je l'amène à la mairie !" (Mme Z, 54 ans, en A, 16 ans d'ancienneté)

\section{Le travail de ménage en situation d'aide : restaurer l'autonomie}

Le ménage, dans ce cadre, est une composante indissociable du travail d'aide. Il relève, en cela, du travail de care, tout particulièrement quand il s'agit de « faire avec $»$; toutefois, cela est vrai à condition que ce travail ne soit pas compromis par l'intervention de tiers qui contribuent, directement ou indirectement, à redéfinir le périmètre de l'activité et surtout il faut poser la question du bénéficiaire final : «Elle vit avec son petit-fils de 17 ans. Quand je fais le repassage, je repasse ses affaires à lui. Mercredi dernier, j'ai repassé treize T-Shirts! Il se change tous les jours, deux fois par jour, elle change les draps facilement tous les trois jours. Le problème c'est que, si je ne le fais pas, c'est elle qui le fera et qui se fatiguera. " (Mme N, 42 ans, en A, 5 ans d'ancienneté)

Ce lien entre le ménage et le travail d'aide, qui renvoie à des manières de faire particulières, se construit au fil de l'expérience, voire des formations. À ce titre, l'expérience versus les premiers pas dans le métier ou encore la formation vs l'absence de formation (sous une forme institutionnalisée ou par les pair-e-s), permettent de rendre compte, à la marge, des positions différentes sur ce point entre intervenantes à domicile. Cela est illustré par une salariée formée qui compare sa pratique avec celle de la collègue qui l'a remplacée auprès de la même personne : "La dame qui a la maladie d'Alzheimer, pendant mon arrêt de travail, elle a eu une aide ménagère qui fait du ménage. Moi, je l'aide à repasser pour la stimuler. " (Mme A., 38 ans, en C, 7 ans d'ancienneté)

Dans ce cadre, le travail d'entretien de la maison, en situation d'aide, diffère du travail de ménage effectué pour un tiers en son absence. Il diffère par exemple du travail des femmes de ménage, des salariées du nettoyage (Benelli, 2007) ou de l'aide à domicile amenées par certains employeurs, associatifs ou privés, à effectuer des heures de ménage dans un domicile en l'absence de ses occupant-e-s ou dans un rapport de subordination (Doniol-Shaw, Lada, Dussuet, 2007). Ce que nous nommons ici le travail de ménage en situation d'aide peut en effet confronter les salariées à des situations « hors-normes »; il s'agit ici moins « d'entretenir le cadre de vie » que de restaurer l'autonomie et la santé des personnes aidées ; l'entretien du domicile tout comme la préparation des repas avec la personne constituent donc, à côté des soins du corps, un enjeu important de ce processus (Doniol-Shaw, 2009). Pour ce faire, les intervenantes élaborent des stratégies afin d'inscrire ce processus comme partie prenante du travail d'aide et de préservation de l'autonomie : «Je vais chez Madame $R$, je vérifie ce qu'elle a pris à déjeuner et je lui fais prendre son bain. Je l'aide à se laver, elle est propre. Je lui passe sa crème aux jambes, je lui chatouille les pieds et je lui fais faire des mouvements d'assouplissement et je la fais chanter. On écoute Chérie FM. Nous faisons le lit ensemble, je fais le ménage de la salle de bains et ensemble nous nettoyons la cuisine. Je lui fais travailler la mémoire, parfois elle oublie de boire, je lui mets des étiquettes partout "penser à boire”. " (Mme P., 49 ans, 5 ans d'ancienneté)

Dans ces nouvelles modalités, les personnes aidées occupent une place centrale, soit parce qu'elles sont invitées à participer à certaines tâches, soit parce que des gestes connexes sont ajoutés, spécifiquement destinés à une personne singulière.

C'est en ce sens qu'il faut comprendre le refus « du ménage » de certaines. Il nous semble en effet que c'est moins le ménage que les conditions/situations du travail de ménage qui sont en jeu. Les salariées s'inscrivent ainsi dans la logique de qualification des métiers de l'aide à domicile, portée par les pouvoirs publics, lorsqu'elles rappellent un des enjeux des tâches ménagères dans le cadre de leur métier et de leurs pratiques : les inscrire comme partie prenante du travail d'aide et de préservation de l'autonomie : «Je ne suis pas contre le ménage. Par exemple, la dame qui a la maladie d'Alzheimer, hier, elle a fait $d u$ repassage avec moi, si vous saviez comme elle 
était contente. C'est ça mon travail, préserver ce qui reste. » (Mme A., 38 ans, en C, 7 ans d'ancienneté)

En termes de pratiques, et à nouveau loin des représentations communes et institutionnelles assimilant le travail de ménage chez soi au travail de ménage en situation d'aide « chez les autres », ce dernier requiert des intervenantes qu'elles ajustent leurs manières de faire ; à cet effet, elles mobilisent certes leurs propres techniques domestiques, mais elles tiennent compte des variantes demandées et du contexte de l'aide à domicile. À cet égard, l'extrait d'entretien suivant est significatif : «À force de travailler avec les personnes âgées, on sait ce qu'il faut faire. Par exemple, je passe toujours la serpillière deux fois, une fois avec la sèche parce qu'elles oublient qu'on est là et elles traversent toute la pièce comme ça. C'est des petites choses qu'on apprend sur le long terme. Au début, je mouillais beaucoup les sols, mais je sais maintenant qu'il ne faut pas mouiller beaucoup les sols. »

Q. «Comment vous l'avez su ? On vous l'a dit?»

«Non, c'est parce que j'ai vu ma personne âgée partie pour traverser la pièce! Il faut penser à tout. Il ne faut rien laisser par terre. On est à deux dans la maison. On n'est pas seule. » (Mme T., 36 ans, en A, 5 ans d'ancienneté)

L'expérience de cette tâche de ménage pousse cette salariée, classée en A, c'est-à-dire sans qualification reconnue, à imaginer une manière différente de réaliser les tâches, qui tienne compte des personnes auxquelles elles sont destinées. À travers cette différenciation de la manière de faire, c'est tout une posture caractéristique du métier qui est apprise ; ce que résume cette autre salariée (qui, elle, a suivi une formation d'auxiliaire de vie sociale) : «C'est le OAA : observer, analyser, agir.»

\section{LES ÉCUEILS DES TRANSFORMATIONS EN COURS SUR LES PRATIQUES}

Or, le «faire » des salariées, tel qu'il s'inscrit dans un dispositif, voire un projet associatif (Weller, 2002), et tel qu'il se traduit en actes pour construire le maintien à domicile, nous semble fragilisé par la dynamique de professionnalisation des salariées et des structures employeuses sous la « convention de professionnalité marchande » (Ribault, 2008). Est ainsi produit un nouveau cadre qui entrave l'exercice du travail d'aide à domicile tel que les intervenantes l'élaborent

\section{L'impact de la réduction des coûts et du vieillissement de la population : mal faire voire faire mal}

En France, l'aide à domicile est soutenue par des aides publiques. La redéfinition de ces aides, et tout particulièrement de l'APA, n'a pas suivi la montée en puissance du nombre des bénéficiaires. Cela a des conséquences sur le travail des aides à domicile, dans un contexte de décentralisation des politiques publiques. Les conseils généraux, qui interviennent à l'échelle des départements, ont ainsi réduit les sommes allouées et donc les temps d'intervention. La réduction du temps d'intervention, alors que le degré de dépendance des personnes restant à leur domicile tend à augmenter, a également induit une fragmentation des interventions. Sur les terrains enquêtés, l'analyse des emplois du temps des salariées et de leur vécu et les entretiens avec les cadres associatifs révèlent un accroissement de la pression temporelle pour les salariées (Doniol-Shaw, Lada, à paraître). Celles-ci doivent faire autant, sinon plus, en moins de temps ; cela a des effets sur leur santé, d'autant plus sensibles qu'elles sont plus âgées (Volkoff et al., 1992 ), ainsi que sur la qualité du travail.

Les personnes les plus dépendantes ont des besoins d'aide matin, midi et soir, et il est de plus en plus fréquent de voir les interventions définies à la demiheure (cas de la toilette ou du repas) voire au quart d'heure (pour un change, la pose de bas de contention, la prise de médicaments ou pour une aide pour aller aux toilettes) ${ }^{6}$. Ces résultats corroborent ceux de recherches en cours, en France, ou menées dans les années 90, aux États-Unis, où le cadre temporel de ces prestations a été standardisé à dix minutes.

Mais, si l'on s'intéresse à ce que signifie « faire un repas », on s'aperçoit qu'il ne s'agit pas seulement de cuisiner. Outre le repas à préparer, cette prestation

\footnotetext{
${ }^{6}$ Certaines associations refusent de telles pratiques au nom des conditions de travail des salariées et de la qualité de l'aide.
} 
s'accompagne de tâches non prescrites : dresser la table, servir le repas, faire la vaisselle, nettoyer l'évier, balayer, nettoyer le sol. De plus, la salle de bains peut nécessiter du rangement et aussi que le lit soit fait. Parallèlement à ces tâches, un travail de discussion s'engage ainsi qu'une aide au déplacement si la personne a des difficultés (ce qui est souvent le cas quand une aide est nécessaire pour les repas). Le travail peut comporter aussi un accompagnement aux toilettes ou un change, ce qu'ignore également la prestation prescrite. Dans les faits, le travail du « faire avec » ou d'accompagnement des personnes, au cœur des objectifs de maintien de leur autonomie, est rendu impossible par les temps impartis.

Ces transformations conduisent par ailleurs à « mal faire » et, pour certaines, à « faire mal », ce qui a un coût particulièrement élevé pour les salariées, certaines allant jusqu'à considérer que ces conditions d'exercice du travail s'apparentent à de la maltraitance : "Quand vous avez une demi-heure pour donner un repas à quelqu'un, c'est une horreur! Parce que vous êtes obligée de bousculer la personne. Moi, j'ai l'impression d'être quelqu'un qui maltraite quelqu'un. Parce que moi, je conçois ça comme de la maltraitance presque. Parce qu'on est obligée de dire : "Bon, écoutez, dépêchez-vous un peu". On est pris par l'heure parce qu'on a quelqu'un à côté. Et ça, je ne le vis pas bien. Ça non. (...) ». (Mme F.B, en C, 18 ans d'ancienneté)

Les essais ou souhaits des salariées, relayées par les associations enquêtées, de participer à l'évaluation des besoins des familles ou personnes aidées à domicile peuvent être appréhendés comme une possibilité de limiter la fragmentation des temps qui se construit en amont de l'association, au moment de l'élaboration des plans d'aide. Certaines associations tentent de se positionner dans l'environnement local, de participer à la territorialisation des politiques sociales en matière de maintien à domicile, ou encore de se faire reconnaître comme acteurs de la prescription.

\section{Les biais de la division du travail d'aide}

En lien avec ce processus de fragmentation des temps, on observe l'imposition de nouvelles formes d'organisation du travail qui reposent sur la division du travail d'aide. Ce processus suit deux voies.
On relève tout d'abord une tendance à diviser le travail d'aide à partir du modèle d'organisation qui s'applique dans un cadre collectif (hôpital ou maison de retraite). Le travail est divisé en tâches spécialisées et standardisées, dont chacune peut faire l'objet d'une prescription temporelle précise, indépendamment du contexte dans lequel elle s'exerce.

Dans ce processus, le ménage devient une prestation en soi pouvant être proposée aux particuliers valides en leur absence et en dehors de tout travail d'aide, alors que les politiques de qualification et de reconnaissance du métier de l'aide à domicile l'avaient inscrit dans le cadre d'une approche globale de l'aide à domicile (Devetter et al., 2008). Cette forme de travail de ménage, en dépit de la volonté de résistance de certains acteurs associatifs au plus près du terrain, apparaît, dans les discours institutionnels, comme une possibilité d'offrir des temps pleins aux salariées et comme un ressort pour diminuer la pénibilité du travail. Or, cette stratégie comporte des risques d'entrave aux processus de professionnalisation de ces métiers, sans pour autant jouer sur la pénibilité du travail. Elle est en effet porteuse d'une érosion des savoirs et savoir-faire des salariées ; en effet, les acquis de la formation ou de l'expérience se perdent et la confiance que l'on peut avoir dans ses capacités en est ébranlée (Leplat, 1975) : «Tout ce que j'ai appris, je ne le mets pas en pratique, et donc j'oublie. » (Mme L., 33 ans, en C, 9 ans d'ancienneté)

La seconde voie empruntée par la division du travail repose sur la distinction entre, d'une part, des tâches qualifiées, s'apparentant à des tâches de soin, qui pourraient être effectuées par les catégories les plus diplômées ; d'autre part, des tâches non qualifiées de ménage, pouvant être confiées à des salariées non formées, ou dont la formation n'est pas reconnue dans l'accord de branche, et évidemment moins bien rémunérées. Cette perspective est elle aussi en contradiction avec l'approche globale portée jusque-là par les pouvoirs publics ainsi qu'avec les pratiques des salariées.

\section{L'isolement au travail}

Un autre biais des transformations en cours réside, sur les terrains enquêtés, dans la construction et l'essor de situations de travail isolées. 
Les salariées travaillent seules au domicile, ce qu'elles apprécient : "Je suis bien dans le métier. Ce qui me plaît, c'est l'autonomie : ils nous font confiance, on ne se sent pas seule, mais on est seule, on gère son travail, sa conscience professionnelle, on n'a personne derrière qui est en train de dire "où tu en es"? Cette autonomie me plaît. Et en même temps, je ne suis pas toute seule, je suis salariée, je ne suis pas indépendante, ça me rassure en même temps pour la sécurité de l'emploi. » (Mme S., 48 ans, en C, 5 ans d'ancienneté)

L'isolement au travail qu'elles connaissent pose en revanche problème. Cet isolement peut prendre différentes formes, souvent conjuguées. Les salariées sont ainsi confrontées à une organisation du travail qui n'apporte pas de réponse à leurs demandes, qui n'assure jamais la rencontre entre les remplaçantes ou encore qui les expose seules à des violences au travail au domicile des particuliers. L'organisation du travail et les lieux du travail (le domicile) construisent en effet un silence organisationnel et un espace qui rendent possibles les violences sexuelles, les mises en position de servitude, le racisme au travail pour les salariées racisées, etc. De même, les salariées sont rarement amenées à se rendre à l'association. Elles y passent au mieux une fois par mois pour retirer des plannings et souvent entre deux prestations. De leur côté, les responsables de secteur, de plus en plus absorbés par l'accroissement des charges administratives et gestionnaires, peinent à éviter l'isolement des salariées dans l'exercice de leur travail.

Ces situations de travail isolées sont produites par l'organisation du travail observée dans les associations ; cependant, elles semblent résulter, directement ou indirectement, des effets des recompositions des financements publics de l'aide à domicile. La disparition des réunions collectives, dans la majorité des associations enquêtées, en est un exemple ${ }^{7}$. C'est particulièrement le cas des associations qui ont réduit le travail d'accompagnement des salariées face à des difficultés financières dues aux recompositions des formes de financements publics de l'aide à domicile.

\footnotetext{
7 Pour combler ce manque, des groupes informels se constituent, au gré de rencontres lors de formation, d'une réunion de fin d'année organisée par l'association ou dans le cadre de leurs relations de voisinage. Ces groupes permettent de « décompresser » mais aussi de préparer des formations ensemble ou de parler du travail.
}

Or, ces réunions représentent des outils importants pour diminuer la pénibilité due à l'isolement au travail, tout en permettant d'élaborer, dans un cadre formel, des savoirs et savoir-faire collectifs ou d'assurer la circulation de « trucs de métier ». Les situations de travail de l'aide à domicile n'offrent en effet pas aux salariées le support que peut constituer un groupe, à l'instar du travail en collectivité, par lequel des savoirs et savoirfaire en pratique peuvent être élaborés.

À ce titre, il nous semble que ces situations de travail isolées constituent l'un des ressorts du turn-over que connait le secteur, en plus des conditions d'emploi et de ce que nous nommons les mises à l'épreuve lors de l'entrée dans le poste : les nouvelles entrantes sont confrontées, dès le premier jour, à des situations extrêmes qui ont en quelque sorte vertu de test : habitats insalubres, troubles importants du comportement (syndrome de Diogène ${ }^{8}$, violences, ...). De telles pratiques relèvent d'une forme de gestion des effectifs que nous relions aux politiques de formation. Face à des difficultés de financement, dans un secteur structuré par un important turn-over, des associations mettraient à l'épreuve les nouvelles entrantes. Elles n'engageraient un processus de formation que pour celles qui ne quittent pas l'association après de telles confrontations. Or, ces pratiques alimenteraient au contraire le turn-over et en seraient l'une des causes.

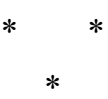

Cette contribution proposait d'analyser les recompositions récentes du travail et des conditions de travail des aides à domicile en milieu associatif, à partir du cas français, dans un contexte de transformation des régimes de care. Nous avons donc tenté de rappeler en quoi les politiques publiques impactent les formes de l'action associative, en participant notamment à redéfinir ses cadres et ses enjeux (Causse et alii, 1998 ; Doniol-Shaw,

\footnotetext{
${ }^{8}$ Le syndrome de Diogène est un syndrome décrit par Clark en 1975 pour caractériser un trouble du comportement de la personne âgée conduisant à des conditions de vie négligées, voire insalubres : négligence parfois extrême de l'hygiène corporelle et domestique ; accumulation d'objets hétéroclites ; déni de son état, associé à une absence de toute honte ; isolement social selon les critères habituellement admis dans sa culture ; refus d'aide concernant cet état, celle-ci étant vécue comme intrusive ; personnalité pré-morbide : soupçonneuse, astucieuse, distante, tendant à déformer la réalité (là encore selon les critères culturels en cours).
} 
Lada, Dussuet, 2007 ; Devetter et alii, 2008). Il s'agissait ainsi de démontrer que l'analyse du travail et de son organisation ne peut faire l'économie, dans le cas de l'aide à domicile, des effets de l'action et des politiques publiques. En effet, le seul périmètre associatif ne suffit pas, tout comme l'échelle organisationnelle ou l'analyse des « bricolages organisationnels » (Coutard, 2001) et des modes de gestion du personnel, dans le cas étudié, à rendre compte des recompositions du travail et des conditions de travail. En ce sens, s'intéresser aux orientations des politiques publiques et au contexte de leur mise en œuvre n'a pas constitué un détour. Une telle orientation constitue au contraire un outil pour se saisir du réel, comme l'a montré la perspective (néo) institutionnaliste. Nous avançons en effet que l'action publique co-organise les formes de mise en emploi et au travail dans l'aide à domicile.
Dans ce cadre, nous avons souligné dans quelle mesure les « nouvelles » prescriptions du travail observées dans les associations, mais aussi les changements de l'organisation formelle du travail qui découlent, tout deux, des orientations les plus récentes des pouvoirs publics et de leur mise en œuvre, sont en profonde contradiction avec le travail réel en situation, tel qu'il se réalise au domicile. Ainsi, le travail d'aide, au cœur de l'activité des aides à domicile, est directement compromis, à travers notamment sa triple expression : le « faire avec », le « faire faire » et le « savoir anticiper pour autrui ». Les conditions de travail et l'espace des qualifications sont également fragilisés ( $c f$. DoniolShaw, dans ce numéro). En d'autres termes, il s'agissait ici de souligner comment les transformations en cours viennent déstabiliser des acquis dans un univers précarisé particulièrement féminisé.

\section{Bibliographie}

Aronson J., Neysmith S. M (1996), "You're not just in there to do the work: Depersonalizing Policies and the exploitation of Home Care Workers'Labor", Gender and Society, $\mathrm{n}^{\circ} 10$ (1), pp. 59-77.

Avril C. (2006), « Le travail des aides à domicile pour personnes âgées : contraintes et savoir-faire », Le Mouvement social, $\mathrm{n}^{\circ} 215$.

Benelli N. (2007), Étude sociologique d'un travail féminin : le nettoyage. Comment tenir le coup dans un sale boulot? Thèse de doctorat, université de Lausanne.

Bettio F., Plantenga J. (2004), "Comparing Care Regimes in Europe", Feminist Economics 10 (1), pp. 85-113.

Causse L., Labruyère C., Fournier C. (1998), Les aides à domicile. Des emplois en plein remueménage, Syros, Paris.

Cloutier E., David H., Ledoux E., Bourdouxe M., Gagnon I., Ouellet F. (2008), "Effects of government policies on the work of home care personnel and their occupational health and safety", Work, 30 (4), pp. 389-402.

Coutard O. (2001), Bricolage organisationnel. Crise des cadres hiérarchiques et innovations dans la gestion des entreprises et des territoires, Paris.

Danet S., Salines E. (2008), « L'état de santé de la population en France. Données du rapport 2007 de suivi des objectifs de la loi de santé publique », Études et résultats, $\mathrm{n}^{\circ} 623$.

Degavre F., Nyssens M. (2008), «L'innovation sociale dans les services d'aide à domicile. Les apports d'une lecture polanyienne et féministe ", Revue française de socio-économie, (2), 2, pp. 79-98.

Devetter F.-X., Jany-Catrice F., Fraisse L, Gardin L., Ribault T. (2008), L'aide à domicile face aux services à la personne, Rapport pour la DIIESES.

Doniol-Shaw G., Lada E. (à paraître), "Work schedules of home care workers for the elderly in France: fragmented work, deteriorating quality, detrimental health impact", Work. 
Doniol-Shaw G. (2009), « L'engagement paradoxal des aides à domicile dans les situations repoussantes », Travailler (22), pp. 27-42

Doniol-Shaw G., Lada E., Dussuet A. (2007), Les parcours professionnels des femmes dans les métiers de l'aide à la personne. Leviers et freins à la qualification et à la promotion. Rapport LATTS. http://www. travail-solidarite.gouv.fr/IMG/pdf/Latts_rapport definitif_21_mars_2008.pdf

Dussuet A. (2005), Travaux de femmes - Enquêtes sur les services à domicile, Paris, L'Harmattan.

Dussuet A., Loiseau D. (2007), « Les services aux familles offerts par les associations : un modèle de service « entre » formel et informel ? ", in Dussuet A., Lauzanas J.M., L'économie sociale entre informel et formel. Paradoxes et innovations, PUR, Rennes, pp. 95-128.

Espagnol P., Lo S.H., Debout C. (2008), «L'allocation personnalisée d'autonomie et la prestation de compensation du handicap », Études et résultats, $\mathrm{n}^{\circ} 666$.

Gaullier X. (1986), «Quel avenir pour le maintien à domicile? ", Revue française des affaires sociales, pp. 9-31.

Guichet F., Hennion A., Paterson F. (2009), Le handicap au quotidien, des expériences d'assistance à domicile, rapport de recherche CNSA/HAS.

Ito R. (2010), « Immigration et travail de care dans une société vieillissante : le cas du Japon » in Falquet J., Hirata H., Kergoat D., Labari B., Le Feuvre N. et Sow F. (éd.), Le sexe de la mondialisation. Genre, classe, race et nouvelle division du travail, Presses de Sciences Po, Paris.

Jany-Catrice F., Puissant F., Ribault T. (2009), « Associations d'aide à domicile : pluralité des héritages, pluralité des professionnalités », Formation Emploi, $\mathrm{n}^{\circ} 107$, pp. 77-91

Jeantet A. (2003), « À votre service ! La relation de service comme rapport social », Sociologie du travail, 45, pp. 191-209.
Jenson J. (2001), « D’un régime de citoyenneté à un autre : la rémunération des soins », Travail, Genre et Sociétés, pp. 43-58.

Lada E. (2009), « Divisions du travail et précarisation de la santé dans le secteur hôtelier en France : de l'action des rapports sociaux de sexe et autres rapports de pouvoir », Travailler, vol. 2, n 22.

Leplat J. (1975), « Développement et dégradation des habiletés dans le travail. Équilibre ou fatigue par le travail ? », Société française de psychologie, Psychologie du travail, ESF.

Le Feuvre N., Martin J. (2001), « Les services de proximité aux ménages : de la solidarité à la précarité de l'emploi féminin », Némésis (3), pp. 299-332.

Marquier R. (2010), « Les intervenantes au domicile des personnes fragilisées en 2008 », Études et résultats, $\mathrm{n}^{\circ} 728$.

Prouteau L. (dir) (2003), Les associations, entre bénévolat et logiques d'entreprise, PUR.

Rocher M., Langevin V. (2005), « D'une maison à l'autre. Risques professionnels et aide à domicile », Audiovisuel-Multimédia, DV 0346, INRS.

Simonazzi A-M. (2008), "Care regimes and national employment models", Cambridge journal of economics, $\mathrm{n}^{\circ} 10$, pp. 1-22.

Ribault T. (2008), « L'aide à domicile face au marché : l'impossible professionnalisation » in Devetter et al., L'aide à domicile face aux services à la personne, Rapport pour la DIIESES.

Volkoff S., Laville A., Maillard M. (1992), « Âges et travail : contraintes, sélection et difficultés chez les 40-50 ans. Une analyse de l'enquête ESTEV », Travail et Emploi (57).

Weller J.-M. (2002), « Stress relationnel et distance au public. De la relation de service à la relation d'aide », Sociologie du travail (1), 44, pp. 75-97. 


\section{Résumé}

\section{Les recompositions du travail d'aide à domicile en France}

Emmanuelle Lada

On observe en France, comme dans d'autres pays du Nord, une mutation de l'organisation sociale, économique et politique de l'aide, du soin et de la dépendance. L'aide à domicile auprès des personnes âgées est particulièrement touchée par ce mouvement. Si ce domaine a connu de nombreuses transformations au cours des précédentes décennies, acteurs et modes de régulation ont subi de profonds changements depuis le début des années 2000, et plus encore à partir de 2005. On s'attachera ici à analyser comment ce processus, porté par les pouvoirs publics, fragilise les conditions de travail et compromet le travail d'aide à domicile, à travers notamment sa triple expression : le "faire avec », le "faire faire » et le «savoir anticiper pour autrui ». Cette analyse des recompositions du travail d'aide à domicile s'appuie sur le cas de structures associatives relevant de l'une des principales fédérations françaises.

\section{Mots clés}

Service aux particuliers, aide à domicile, professionnalisation, conditions de travail

Journal of Economic Literature : L 84, J 24 